\title{
Thoracic endovascular aortic repair for type B intramural hematoma
}

\author{
A. Claire Watkins ${ }^{1}$, Andreas R. de Biasi ${ }^{1}$, Shinichi Iwakoshi ${ }^{1}$, Yukihisa Ogawa ${ }^{1}$, Michael D. Dake ${ }^{2}$ \\ ${ }^{1}$ Department of Cardiothoracic Surgery, Stanford University School of Medicine, Stanford, CA, USA; ${ }^{2}$ University of Arizona Health Sciences, \\ Tucson, AZ, USA \\ Correspondence to: Michael D. Dake, MD. Drachman Hall B-207, 1295 North Martin Ave, PO Box 210202, Tucson, AZ 85721, USA. \\ Email: mddake@stanford.edu.
}

Submitted Mar 13, 2019. Accepted for publication Jun 03, 2019.

doi: 10.21037/acs.2019.06.06

View this article at: http://dx.doi.org/10.21037/acs.2019.06.06

\section{Clinical vignette}

A 66-year-old woman presented with sudden onset sharp back pain. Past medical history included hypertension with medication non-compliance, diabetes, hyperlipidemia, obstructive sleep apnea and former tobacco use. She was hypotensive upon arrival with a hemoglobin of $8.1 \mathrm{~g} / \mathrm{dL}$, but hemodynamically stabilized with fluid resuscitation. She reported no shortness of breath, gastrointestinal or neurologic symptoms, or trauma. A CT scan revealed extensive descending aortic intramural hematoma (IMH) with posterior hemomediastinum and bilateral hemathoraces. No intimal dissection flap was evident. She underwent emergent thoracic endovascular aortic repair (TEVAR) for type B aortic IMH with rupture.

\section{Surgical techniques}

\section{Preparation}

Prior to the procedure, aortic stent graft landing zones at the level of the left subclavian artery and celiac artery had diameters of 29 and $25 \mathrm{~mm}$, respectively. TEVAR was done under general anesthesia in a hybrid operating room with a fixed imaging system.

\section{Exposition}

Surgical exposure of the right femoral artery was done through a $3-\mathrm{cm}$, longitudinal incision. Percutaneous femoral arterial access on the contralateral side was obtained under ultrasound guidance and a 5-Fr sheath and pigtail catheter were introduced for angiographic imaging.

\section{Operation}

A 7-Fr sheath was placed directly in the surgically exposed right common femoral artery. The pigtail was advanced to the ascending aorta. Initial aortography demonstrated no ongoing contrast extravasation or site suspicious for rupture. Subsequently, a guidewire and catheter were advanced to the ascending aorta from the right side. Following exchange for a curved Lunderquist guidewire (Cook Medical, Bloomington, IN), a 22-Fr DrySeal sheath (WL Gore, Flagstaff, AZ, USA) was advanced to the abdominal aorta. Aortography demonstrated the origin of the celiac artery and a pair of large spinal arteries. A $28 \mathrm{~mm} \times 150 \mathrm{~mm}$ Gore C-TAG endograft was deployed immediately above this landmark. Arch aortography then showed the origin of the left subclavian artery and a $31 \mathrm{~mm} \times 150 \mathrm{~mm}$ Gore C-TAG endograft was deployed immediately distal to the left subclavian artery with $5-7 \mathrm{~cm}$ of overlap with the distal stent graft. No balloon molding was done. Completion aortography demonstrated good positioning of the endografts without endoleak or other complication.

\section{Completion}

The patient made an uneventful recovery. Surveillance CT imaging at 1 and 3 months revealed no endoleak and continued remodeling. 


\section{Comment}

\section{Clinical results}

Although distinct from the classic type B dissection with true and false lumens, type B IMH carries a similar risk for aortic rupture. Comprising approximately $10 \%$ of descending aortic pathology, it is unknown whether IMH arises from rupture of vasa vasorum in the aortic media or from unseen tears in the intima. While $58.5 \%$ of type B IMH may stabilize or resolve with conservative management, $48.7 \%$ will progress to aneurysm, classic dissection or rupture $(1,2)$. It is recommended that symptomatic IMH and penetrating aortic ulcer (PAU) be treated similarly to classic type B dissection $(1,3)$. In addition to the classic indications for intervention of rupture, malperfusion, maximal diameter $>5.5 \mathrm{~cm}$, rapid expansion or persistent hypertension or pain, the presence of ulcer-like projections (ULP) or PAU also warrants TEVAR due to a high likelihood of rupture. Additionally, TEVAR is generally recommended over open surgical repair for symptomatic, anatomically suitable cases without infection or connective tissue disease. This case illustrates a symptomatic type B IMH complicated by rupture without an obvious entry tear. In this situation, as with most ruptures in the descending thoracic aorta, stentgraft coverage from the left subclavian to the celiac artery is indicated. The thickness of IMH in this case was $18 \mathrm{~mm}$. It has been shown that the thickness of IMH correlates with complications (4) and some advocate for intervention on all $\mathrm{IMH}>10 \mathrm{~mm}$ (5).

Results for type B IMH treated either medically or with TEVAR are generally favorable. In a recent review, uncomplicated type B IMH treated medically had an acute mortality rate of 3.4\%, 3-year aortic event rate of 5.4\% but with a late intervention rate approaching $50 \%$. Acute mortality among complicated type B IMH was $16 \%$ with open surgical repair and $4.6 \%$ with TEVAR (1). A study of 41 patients showed that freedom from aortic-related mortality or reintervention was $92 \%$ for complicated type B IMH treated with TEVAR (2). Another retrospective study comparing medical management to immediate TEVAR for symptomatic type B IMH demonstrated a remarkable improvement in freedom from aortic-related adverse events or mortality at 36 months with TEVAR (17\% vs. $72 \%$ ) (4). TEVAR for IMH has been shown to promote aortic remodeling, with significant true lumen expansion, IMH resolution and a decrease in the total aortic diameter after TEVAR (5). As with classic type B dissection, symptomatic type B IMH treated with TEVAR can prolong survival, reduce aortic related complications and promote favorable aortic remodeling.

\section{Advantages and caveats}

In the setting of rupture, expeditious stent-graft repair is paramount. As with classic type B dissections, repair should not oversize the stent-graft more than $10 \%$ greater than the native aorta. Proximal and distal landing zones should consist of normal appearing aorta, which can be difficult to obtain with extensive IMH. In this case, the proximal and distal landing zones had 8 and $4 \mathrm{~mm}$ of IMH respectively, but were distant from both the rupture and area of maximal IMH. In the setting of rupture or hemodynamic instability, it may be wise to choose a simpler, zone 3 proximal neck, despite some IMH at the landing zones. Additionally, balloon angioplasty of IMH is ill-advised as it can lead to retrograde type A dissection or a stent-graft induced new entry tear as reported in classic type B dissection. Intravascular ultrasound (IVUS) can provide important verification of true lumen access in type B dissection. In non-ruptured type B IMH, IVUS may allow visualization of microtears. Regardless, repair should center on both the location of any microtears, PAU or ULPs and the maximal area of thickness of IMH. With no obvious intimal tear on CT and contained rupture, IVUS would have served no purpose in this case. We implanted the smaller, distal stent graft piece first and then the larger-diameter proximal device. In a classic type B dissection, this poses risk for significant, temporary false lumen pressurization, depending on the location of re-entry tears. With no obvious entry tears, the standard sequence of smaller diameter stent-graft implantation first in situations with differential landing zone diameters appears to be preferred.

\section{Acknowledgments}

None.

\section{Footnote}

Conflicts of Interest: The authors have no conflicts of interest to declare.

\section{References}

1. Evangelista A, Czerny M, Nienaber C, et al. Interdisciplinary expert consensus on management of type 
B intramural haematoma and penetrating aortic ulcer. Eur J Cardiothorac Surg 2015;47:209-17.

2. Piffaretti G, Lomazzi C, Benedetto F, et al. Best Medical Treatment and Selective Stent-GraftRepair for Acute Type B Aortic Intramural Hematoma. Semin Thorac Cardiovasc Surg 2018;30:279-87.

3. Hiratzka LF, Bakris GL, Beckman JA, et al. 2010 ACCF/AHA/AATS/ACR/ASA/SCA/SCAI/SIR/STS/ SVM Guidelines for the diagnosis and management of patients with thoracic aortic disease. A Report of the American College of Cardiology Foundation/American Heart Association Task Force on Practice Guidelines, American Association for Thoracic Surgery, American

Cite this article as: Watkins $\mathrm{AC}$, de Biasi AR, Iwakoshi S, Ogawa Y, Dake MD. Thoracic endovascular aortic repair for type B intramural hematoma. Ann Cardiothorac Surg 2019;8(4):509-511. doi: 10.21037/acs.2019.06.06
College of Radiology,American Stroke Association, Society of Cardiovascular Anesthesiologists, Society for Cardiovascular Angiography and Interventions, Society of Interventional Radiology, Society of Thoracic Surgeons, and Society for Vascular Medicine. J Am Coll Cardiol 2010;5 :e27-129.

4. Mesar T, Lin MJ, Kabir I, et al. Type B Aortic Intramural Hematoma: To TEVAR or not to TEVAR? J Vasc Surg 2019;69:e4.

5. Lavingia KS, Ahanchi SS, Redlinger RE, et al. Aortic remodeling after thoracic endovascular aortic repair for intramural hematoma. J Vasc Surg 2014;60:929-35; discussion 935-6. 